\title{
The Adomian Decomposition Method with Discretization for Second Order Initial Value Problems
}

\author{
Dagnachew Mengstie Tefera and Awoke Andargie
}

\begin{abstract}
In this paper, Adomian Decomposition Method with Discretization (ADMD) is applied to solve both linear and nonlinear initial value problems (IVP). Comparison with Adomian Decomposition Method (ADM) is presented. To illustrate the efficiency and accuracy of the method, five examples are considered. The result shows that ADMD is more efficient and accurate than ADM.
\end{abstract}

Index Terms-Decomposition method, Adomian polynomial, initial value problems, infinite series.

\section{INTRODUCTION}

A DOMIAN decomposition method (ADM) [1], [2], which was first introduced by the American Physicist George Adomian, has been used to solve effectively and easily a large class of linear and nonlinear ordinary and partial differential equations. This method generates a solution in the form of a series whose terms are determined by a recursive relationship using the Adomian polynomials [3], [4].

The non-linear problems are solved easily and elegantly without linearizing the problem by using ADM. It also avoids the linearization, perturbation and discretization unlike other classical techniques [5], [6]. The main advantage of this method is that it can be applied directly to all types of differential and integral equations, either linear or non-linear, homogeneous or inhomogeneous, with constant or variable coefficients. Another important advantage is that, the method is capable of greatly reducing the size of computational works while still maintaining high accuracy of the numerical solution [7]. The decomposition method produced reliable results with less iteration, than the Taylor series method and the RungeKutta methods [8], [9]. The convergence of the ADM has been investigated by a number of authors [10], [11].

In this study, we applied the ADMD to solve the initial value problem of linear and non-linear second order ODE. ADMD differs from $\mathrm{ADM}$, i.e. it divides the interval into a finite number of subintervals and for each subinterval it generates a solution in the form of a series whose terms are determined by a recursive relationship using the Adomian polynomials. The results show that the ADMD is more accurate and suitable solution than the ADM. Also, if the order of the differential equation increases, the solution using ADMD is better than ADM.

Manuscript received April 25, 2019; accepted July 29, 2019.

D.M. Tefera is with the Department of Mathematics, Debre Tabor University, Debre Tabor, Ethiopia. E-mail: dagnmeng@yahoo.com

A. Andargie is with the Department of Mathematics, Bahir Dar University, Bahir Dar, Ethiopia. E-mail: awoke248@yahoo.com

\section{Adomian Decomposition Method}

Consider the following second order ordinary differential equation

$$
L y+R y+N y=g(x),
$$

where $L=\frac{d^{2}}{d x^{2}}$ is a linear operator, $R$ is the remaining linear lower order derivative, $N$ is a nonlinear operator and $g$ is any function. Integrating (1) yields

$$
y=y(0)+x y^{\prime}(0)+L^{-1} g-L^{-1} R y-L^{-1} N y,
$$

for the initial value problem, where $L^{-1}(\cdot)=\int_{0}^{x} \int_{0}^{x}(\cdot) d t d t$.

The Adomian Decomposition Method assumes that the unknown function $y$ can be expressed by infinite series of the form

$$
y=\operatorname{sum}_{n=0}^{\infty} y_{n} .
$$

The ADM assumes that the nonlinear operator $N(y)$ can be decomposed by an infinite series of polynomial given by

$$
N(y)=\sum_{n=0}^{\infty} A_{n},
$$

where $A_{n}$ are the Adomian's polynomials defined as $A_{n}=$ $A_{n}\left(y_{0}, y_{1}, y_{2}, \ldots, y_{n}\right)$. Substituting (3) and (4) into equation (2) and using the fact that $R$ is a linear operator, we obtain

$$
\sum_{n=0}^{\infty} y_{n}=y(0)+x y^{\prime}(0)+L^{-1} g-L^{-1}\left(\sum_{n=0}^{\infty} R\left(y_{n}\right)\right)-L^{-1}\left(\sum_{n=0}^{\infty} A_{n}\right) \text {. }
$$

Therefore, the formal recurrence algorithm could be defined by

$$
\left.\begin{array}{l}
y_{0}=y(0)+x y^{\prime}(0)+L^{-1}(g) \\
y_{1}=-L^{-1}\left(R\left(y_{0}\right)\right)-L^{-1}\left(A_{0}\left(y_{0}\right)\right) \\
y_{2}=-L^{-1}\left(R\left(y_{1}\right)\right)-L^{-1}\left(A_{1}\left(y_{0}, y_{1}\right)\right) \\
\vdots \\
y_{n+1}=-L^{-1}\left(R\left(y_{n}\right)\right)-L^{-1}\left(A_{n}\left(y_{0}, y_{1}, y_{2}, \ldots, y_{n}\right)\right)
\end{array}\right\}
$$

The Adomian polynomial $A_{n}$ was first introduced by Adomian himself. It was defined via the following general formula

$$
A_{n}=\frac{1}{n !} \frac{d^{n}}{d \lambda^{n}}\left(N\left(\sum_{k=0}^{\infty} y_{k} \lambda^{k}\right)\right)_{\lambda=0} \quad n=0,1,2, \ldots
$$

The first few Adomian polynomials are

$$
\left.\begin{array}{l}
A_{0}=N\left(y_{0}\right) \\
A_{1}=N^{\prime}\left(y_{0}\right) y_{1} \\
A_{2}=N^{\prime}\left(y_{0}\right) y_{2}+N^{\prime \prime}\left(y_{0}\right) \frac{y_{1}^{2}}{2 !} \\
A_{3}=N^{\prime}\left(y_{0}\right) y_{3}+N^{\prime \prime}\left(y_{0}\right) y_{1} y_{2}+N^{\prime \prime \prime}\left(y_{0}\right) \frac{y_{1}^{3}}{3 !}
\end{array}\right\}
$$




\section{Adomian Decomposition Method with DISCRETIZATION}

Consider the following second-order ordinary differential equation

$$
L y+R y+N y=g(x)
$$

where $L=\frac{d^{2}}{d x^{2}}$ is a linear operator, $R$ is the remaining linear lower order derivative, $N$ is a nonlinear operator and $g$ is any function.

ADMD divides the working interval $[0,1]$ into $m$ equal subintervals with $h=x_{i+1}-x_{i}$ for $i=0,1,2, \ldots, m-1$ where $x_{0}=0$ and $x_{m}=1$. Integrating (1), on the $(i+1)^{t h}$ interval on $\left[x_{i}, x\right]$ for $i=0,1,2, \ldots, m-1$

$$
y_{i+1}=y_{i}\left(x_{i}\right)+y_{i}^{\prime}(0)\left(x-x_{i}\right)+L^{-1} g-L^{-1} R y_{i}-L^{-1} N y_{i}
$$

where $y_{i+1}\left(x_{i}\right)=y_{i}\left(x_{i}\right)$ and $y_{i+1}^{\prime}\left(x_{i}\right)=y_{i}^{\prime}\left(x_{i}\right)$ and $L^{-1}(\cdot)=$ $\int_{x_{i}}^{x} \int_{x_{i}}^{x}(\cdot) d t d t$.

ADMD assumes that the unknown function can be expressed by infinite series

$$
y_{i+1}=\sum_{n=0}^{\infty} y_{n, i} \quad \text { for } i=0,1,2, \ldots, m-1
$$

and the ADMD assumes that the nonlinear operator $N\left(y_{i}\right)$ can be decomposed by an infinite series of polynomial given by

$$
N\left(y_{i}\right)=\sum_{n=0}^{\infty} A_{n, i} \quad \text { for } i=0,1,2, \ldots, m-1
$$

where $A_{n, i}$ are the Adomian's polynomials defined as $A_{n, i}=$ $A_{n, i}\left(y_{0, i}, y_{1, i}, y_{2, i}, \ldots, y_{n, i}\right)$. Substituting (3) and (4) into equation (2) and using the fact that $R$ is a linear operator, we obtain

$$
\begin{aligned}
& \sum_{n=0}^{\infty} y_{n, i}=y\left(x_{i}\right)+y_{i}^{\prime}\left(x_{i}\right)\left(x-x_{i}\right)+L^{-1} g-L^{-1}\left(\sum_{n=0}^{\infty} R\left(y_{n, i}\right)\right) \\
& -L^{-1}\left(\sum_{n=0}^{\infty} A_{n, i}\right)
\end{aligned}
$$

for $i=0,1,2, \ldots, m-1$.

Therefore, the formal recurrence algorithm could be defined by

$$
\left.\begin{array}{l}
y_{0, i}=y\left(x_{i}\right)+y^{\prime}\left(x_{i}\right)\left(x-x_{i}\right)+L^{-1}(g) \\
y_{1, i}=-L^{-1}\left(R\left(y_{0, i}\right)\right)-L^{-1}\left(A_{0, i}\left(y_{0, i}\right)\right) \\
y_{2, i}=-L^{-1}\left(R\left(y_{1, i}\right)\right)-L^{-1}\left(A_{1, i}\left(y_{0, i}, y_{1, i}\right)\right) \\
\vdots \\
y_{n+1, i}=-L^{-1}\left(R\left(y_{n, i}\right)\right)-L^{-1}\left(A_{n, i}\left(y_{0, i}, y_{1, i}, y_{2, i}, \ldots, y_{n, i}\right)\right)
\end{array}\right\}
$$

The Adomian polynomial $A_{n, i}$ was first introduced by Adomian himself. It was defined via the following general formula

$$
A_{n, i}=\frac{1}{n !} \frac{d^{n}}{d \lambda^{n}}\left(N\left(\sum_{k=0}^{\infty} y_{k, i} \lambda^{k}\right)\right)_{\lambda=0} \quad n=0,1,2, \ldots
$$

for $i=0,1,2, \ldots, m-1$.

The first few Adomian polynomials are

$$
\left.\begin{array}{l}
A_{0, i}=N\left(y_{0, i}\right) \\
A_{1, i}=N^{\prime}\left(y_{0, i}\right) y_{1, i} \\
A_{2, i}=N^{\prime}\left(y_{0, i}\right) y_{2, i}+N^{\prime \prime}\left(y_{0, i}\right) \frac{y_{1, i}^{2}}{2 !} \\
A_{3, i}=N^{\prime}\left(y_{0, i}\right) y_{3, i}+N^{\prime \prime}\left(y_{0, i}\right) y_{1, i} y_{2, i}+N^{\prime \prime \prime}\left(y_{0, i}\right) \frac{y_{1, i}^{3}}{3 !}
\end{array}\right\}
$$

a) Example 1: Consider the following second order linear ordinary differential equation

$$
y^{\prime \prime}-y=0 \text { with } y(0)=0 \text { and } y^{\prime}(0)=1
$$

The exact solution is $y=\sin x$. By using linear operator $L$, (17) can be written as

$$
L y-y=0 \text { with } y(0)=0 \text { and } y^{\prime}(0)=1
$$

By using ADM, we apply $L^{-1}$ to both sides of (18) and using initial condition, we obtain

$$
y=y(0)+x y^{\prime}(0)+L^{-1}(y)
$$

Upon using the decomposition series for the solution

$$
y=\sum_{n=0}^{\infty} y_{n}=x+L^{-1}\left(\sum_{n=0}^{\infty} y_{n}\right)
$$

This leads to the recursive relation

$$
y_{0}=x, \quad y_{n+1}=\int_{0}^{x} \int_{0}^{x} y_{n} d t d t
$$

The first five terms of the series are

$$
y_{0}=x, y_{1}=\frac{x^{3}}{6}, y_{2}=\frac{x^{5}}{120}, y_{3}=\frac{x^{7}}{5040}, y_{4}=\frac{x^{9}}{362880}
$$

More components in the decomposition series can be calculated to enhance the accuracy of the approximation. By computing five terms of the solution series, we obtain

$$
y=\sum_{n=0}^{4} y_{n}=x+\frac{x^{3}}{6}+\frac{x^{5}}{120}+\frac{x^{7}}{5040}+\frac{x^{9}}{362880}
$$

for $0 \leq x \leq 1$.

By using ADMD, we divide the interval $[0,1]$ into $m$ subintervals $h=x_{i+1}-x_{i}$ for $i=0,1,2, \ldots, m-1$ where $x_{0}=0$ and $x_{m}=1$. Applying $L^{-1}$ to both sides in (18) and using the initial condition, we obtain

$$
y_{i+1}=y_{i}\left(x_{i}\right)+y_{i}^{\prime}\left(x_{i}\right)\left(x-x_{i}\right)+L^{-1}\left(y_{i}\right)
$$

Upon using the decomposition series for the solution is

$$
y_{i+1}=\sum_{n=0}^{\infty} y_{n, i}=y_{i}\left(x_{i}\right)+y_{i}^{\prime}\left(x-x_{i}\right)+L^{-1}\left(\sum_{n=0}^{\infty} y_{n, i}\right)
$$

for $i=0,1,2, \ldots, m-1$.

This leads to the following recursive equation

$$
\begin{aligned}
y_{0, i} & =y_{i}\left(x_{i}\right)_{i}^{\prime}\left(x-x_{i}\right) \\
y_{n+1, i} & =\int_{x_{i}}^{x} \int_{x_{i}}^{x} y_{n, i} d t d t \\
& \text { where } y_{i+1}\left(x_{i}\right)=y_{i}\left(x_{i}\right) \text { and } y_{i+1}^{\prime}\left(x_{i}\right)=y_{i}^{\prime}\left(x_{i}\right) .
\end{aligned}
$$

Thus on the first interval, we select $\alpha=0$ to obtain

$$
\begin{aligned}
y_{0,0} & =x \\
y_{n+1,0} & =\int_{0}^{x} \int_{0}^{x} y_{n, 0} d t d t \text { where } n=0,1,2,3,4 \\
y_{1} & =\sum_{n=0}^{4} y_{n, 0}
\end{aligned}
$$


TABLE I: Error values for the approximation solution of ADM and ADMD

\begin{tabular}{c||c|c|c}
\hline \multicolumn{1}{c||}{$x$} & \multirow{2}{*}{ Error ADM } & \multicolumn{2}{c}{ Error ADMD } \\
\cline { 3 - 4 } & & $h=0.25$ & $h=0.1$ \\
\hline \hline 0 & 0 & 0 & 0 \\
0.10 & $6.9389 \mathrm{e}-17$ & $6.9389 \mathrm{e}-17$ & $6.9389 \mathrm{e}-17$ \\
0.20 & $5.2736 \mathrm{e}-16$ & $5.2736 \mathrm{e}-16$ & $2.7756 \mathrm{e}-17$ \\
0.30 & $4.4464 \mathrm{e}-14$ & $1.9151 \mathrm{e}-14$ & $1.5846 \mathrm{e}-09$ \\
0.40 & $1.0518 \mathrm{e}-12$ & $4.6074 \mathrm{e}-14$ & $2.2204 \mathrm{e}-16$ \\
0.50 & $1.2252 \mathrm{e}-11$ & $1.4522 \mathrm{e}-13$ & $5.5511 \mathrm{e}-16$ \\
0.60 & $9.1098 \mathrm{e}-11$ & $4.6640 \mathrm{e}-13$ & $1.6072 \mathrm{e}-12$ \\
0.70 & $4.9692 \mathrm{e}-10$ & $6.4748 \mathrm{e}-13$ & $2.9088 \mathrm{e}-14$ \\
0.80 & $2.1608 \mathrm{e}-09$ & $1.5594 \mathrm{e}-12$ & $1.7764 \mathrm{e}-15$ \\
0.90 & $7.9026 \mathrm{e}-09$ & $2.4882 \mathrm{e}-12$ & $2.6645 \mathrm{e}-15$ \\
1.00 & $2.5213 \mathrm{e}-08$ & $3.6633 \mathrm{e}-12$ & $4.2188 \mathrm{e}-15$ \\
\hline
\end{tabular}

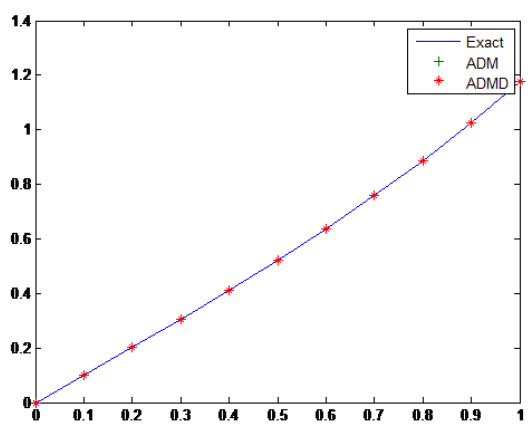

Fig. 1: Comparison between the exact solution, solution obtained from ADM and solution obtained from ADMD for $h=0.1$

Similarly, for $i=1,2, \ldots, m-1$

$$
\begin{aligned}
y_{0, i} & =y_{i}\left(x_{i}\right)+y_{i}^{\prime}\left(x_{i}\right)\left(x-x_{i}\right) \\
y_{n+1, i} & =\int_{x_{i}}^{x} \int_{x_{i}}^{x} y_{n, i} d t d t \\
& \text { where } y_{i+1}\left(x_{i}\right)=y_{i}\left(x_{i}\right) \text { and } y_{i+1}^{\prime}\left(x_{i}\right)=y_{i}^{\prime}\left(x_{i}\right) \\
y_{i+1} & =\sum_{n=0}^{4} y_{n, i}
\end{aligned}
$$

The result of ADM and ADMD are illustrated in Table I with five terms of the series at $h=0.1$ and $h=0.25$. Clearly, ADMD is more accurate than ADM and the accuracy of the ADMD can be increased either by choosing a small step size or by adding more terms of the solution series. problem

b) Example 2: Consider the Bratu-type initial value

$$
y^{\prime \prime}-\lambda e^{y}=0 \text { with } y(0)=y^{\prime}(0)=0
$$

The exact solutions are

$$
\left\{\begin{aligned}
y=\ln \left(1+\tan \left(\frac{x \sqrt{2}}{2}\right)^{2}\right), & \text { if } \lambda=1 \\
y=-2 \ln (\cos x), & \text { if } \lambda=2
\end{aligned}\right.
$$

By using linear operator $L,(17)$ can be written as

$$
\text { Ly- } \lambda e^{y}=0 \text { with } y(0)=y^{\prime}(0)
$$

By applying $L^{-1}$ to both sides in (18) and by using the initial condition, we obtain

$$
y=\lambda L^{-1}\left(e^{y}\right)
$$

By using the ADM, we assume $y$ and nonlinear term as infinite series given by

$$
\begin{aligned}
y & =\sum_{n=0}^{\infty} y_{n}=\lambda L^{-1}\left(\sum_{n=0}^{\infty} A_{n}\right) \\
y_{0} & =0 \\
y_{n+1} & =\lambda L^{-1}\left(A_{n}\right)=\lambda \int_{0}^{x} \int_{0}^{x} A_{n} d t d t \quad \text { for } n=0,1,2, \ldots
\end{aligned}
$$

where $A_{n}$ is Adomian polynomial representation for the nonlinear term $e^{y}$ gives

$$
A_{0}=1, A_{1}=y_{1}, A_{2}=y_{2}+\frac{1}{2} y_{1}^{2}, A_{3}=y_{3}+y_{1} y_{2}+\frac{1}{6} y_{1}, \ldots
$$

By using ADMD, We divide the interval $[0,1]$ into $m$ subintervals $h=x_{i+1}-x_{i}$ for $i=0,1,2, \ldots, m-1$ where $x_{0}=0$ and $x_{m}=1$. We apply $L^{-1}$ to both sides in (20) and by using the initial condition, we obtain

$$
y_{i+1}=y_{i}\left(x_{i}\right)+y_{i}^{\prime}\left(x_{i}\right)\left(x-x_{i}\right)+\lambda L^{-1}\left(e^{y}\right)
$$

Using the decomposition method assumes $y$ and nonlinear term as infinite series, given by

$$
y_{i+1}=\sum_{n=0}^{\infty} y_{n, i}=y_{i}\left(x_{i}\right)+y_{i}^{\prime}\left(x_{i}\right)\left(x-x_{i}\right)+\lambda L^{-1}\left(\sum_{n=0}^{\infty} A_{n}\right)
$$

for $i=0,1,2, \ldots, m-1$. This leads to the following recursive relation

$$
\begin{aligned}
y_{0, i} & =y_{i}\left(x_{i}\right)+y_{i}^{\prime}\left(x_{i}\right)\left(x-x_{i}\right) \\
y_{n+1, i} & =\lambda \int_{x_{i}}^{x} \int_{x_{i}}^{x} A_{n, i} d t d t \\
& \text { where } y_{i+1}\left(x_{i}\right)=y_{i}\left(x_{i}\right) \text { and } y_{i+1}^{\prime}\left(x_{i}\right)=y_{i}^{\prime}\left(x_{i}\right)
\end{aligned}
$$

where $A_{n, i}$ is the Adomian polynomial representation for the nonlinear term $e^{y}$, gives

$$
A_{n, i}=\frac{1}{n !} \frac{d^{n}}{d \lambda^{n}}\left[N\left(\sum_{k=0}^{\infty} y_{k, i} \lambda^{k}\right)\right]_{\lambda=0} \quad n=0,1,2, \ldots
$$

for $i=0,1,2, \ldots, m-1$. The result of ADM and ADMD are illustrated in Table 2 below with five terms of the series for $\lambda=1$ and $\lambda=2$ at $h=0.25$. Clearly, ADMD is more accurate than ADM and the accuracy of the ADM and ADMD increased for Bratu's equation if $\lambda$ is small and choosing a small step size or by adding more terms of the solution series.

c) Example 3: Consider the following non-homogeneous linear differential equation

$$
y^{\prime \prime}-y=x \text { with } y(0)=1 \text { and } y^{\prime}(0)=0
$$

The exact solution is $y(x)=e^{x}-x$. The result of ADM and ADMD are illustrated in Table III below with five terms of the series at $h=0.1$ and $h=0.25$. Clearly, ADMD is more accurate than ADM. Figure 3 shows the comparison between $\mathrm{ADM}$ and ADMD at $h=0.1$ and $h=0.25$. 
TABLE II: Error values for the approximate solution of ADM and ADMD

\begin{tabular}{c||c|c|c|c}
\hline \multicolumn{1}{c||}{$x$} & \multicolumn{2}{c|}{$\lambda=1$} & \multicolumn{2}{c}{$\lambda=2$} \\
\cline { 2 - 5 } & Error ADM & Error ADMD & Error ADM & Error ADMD \\
\hline \hline 0.0 & $0.0 \mathrm{e}+00$ & $0.0 \mathrm{e}+00$ & $0.0 \mathrm{e}+00$ & $0.0 \mathrm{e}+00$ \\
0.1 & $1.4 \mathrm{e}-14$ & $1.4 \mathrm{e}-14$ & $4.4 \mathrm{e}-13$ & $4.4 \mathrm{e}-13$ \\
0.2 & $1.4 \mathrm{e}-11$ & $1.4 \mathrm{e}-11$ & $4.5 \mathrm{e}-10$ & $4.5 \mathrm{e}-10$ \\
0.3 & $8.2 \mathrm{e}-10$ & $3.8 \mathrm{e}-10$ & $2.7 \mathrm{e}-08$ & $1.3 \mathrm{e}-08$ \\
0.4 & $1.5 \mathrm{e}-08$ & $9.4 \mathrm{e}-10$ & $4.8 \mathrm{e}-07$ & $3.0 \mathrm{e}-08$ \\
0.5 & $1.4 \mathrm{e}-07$ & $1.6 \mathrm{e}-09$ & $4.7 \mathrm{e}-06$ & $5.6 \mathrm{e}-08$ \\
\hline
\end{tabular}

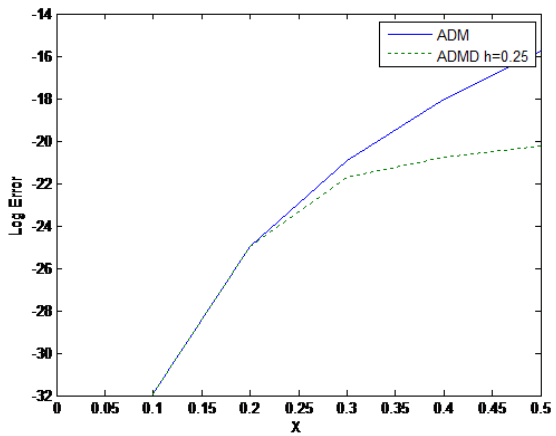

Fig. 2: Comparison between exact solution, solution obtained using ADM and solution obtained using ADMD for $h=0.25$ and $\lambda=1$ where Log Error is the logarithm value of absolute error.

TABLE III: Error values for the approximation solution of $\mathrm{ADM}$ and ADMD

\begin{tabular}{c||c|c|c}
\hline \multirow{2}{*}{$x$} & \multirow{2}{*}{ Error ADM } & \multicolumn{2}{|c}{ Error ADMD } \\
\cline { 3 - 4 } & & $h=0.25$ & $h=0.1$ \\
\hline \hline 0 & 0 & 0 & 0 \\
0.1 & $0.00 \mathrm{e}+00$ & $0.00 \mathrm{e}+00$ & $0.00 \mathrm{e}+00$ \\
0.2 & $2.82 \mathrm{e}-14$ & $2.82 \mathrm{e}-14$ & $4.44 \mathrm{e}-16$ \\
0.3 & $1.63 \mathrm{e}-12$ & $7.89 \mathrm{e}-13$ & $6.66 \mathrm{e}-16$ \\
0.4 & $2.89 \mathrm{e}-11$ & $1.85 \mathrm{e}-12$ & $2.00 \mathrm{e}-15$ \\
0.5 & $2.70 \mathrm{e}-10$ & $3.20 \mathrm{e}-12$ & $3.11 \mathrm{e}-15$ \\
\hline
\end{tabular}

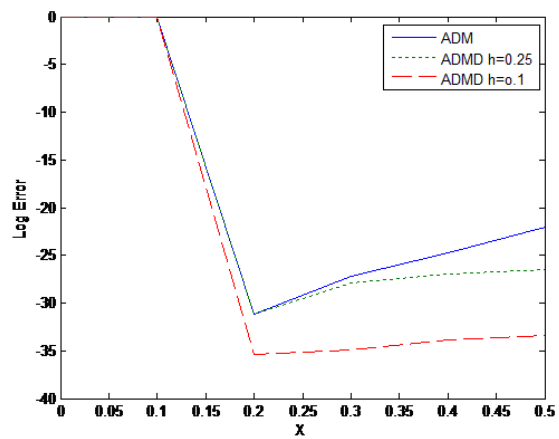

Fig. 3: Comparison between exact solution, solution obtained using ADM and solution obtained using ADMD for $h=0.25$ and $h=0.1$ where Log Error is the logarithm value of absolute error.
TABLE IV: Error values for the approximate solution of ADM and ADMD

\begin{tabular}{c||c|c}
\hline$x$ & Error ADM & Error ADMD for $h=0.25$ \\
\hline \hline 0 & 0 & 0 \\
0.1 & $1.56 \mathrm{e}-10$ & $1.56 \mathrm{e}-10$ \\
0.2 & $1.16 \mathrm{e}-07$ & $1.16 \mathrm{e}-07$ \\
0.3 & $4.86 \mathrm{e}-06$ & $2.63 \mathrm{e}-06$ \\
0.4 & $6.25 \mathrm{e}-05$ & $6.21 \mathrm{e}-06$ \\
0.5 & $4.20 \mathrm{e}-04$ & $1.02 \mathrm{e}-05$ \\
\hline
\end{tabular}

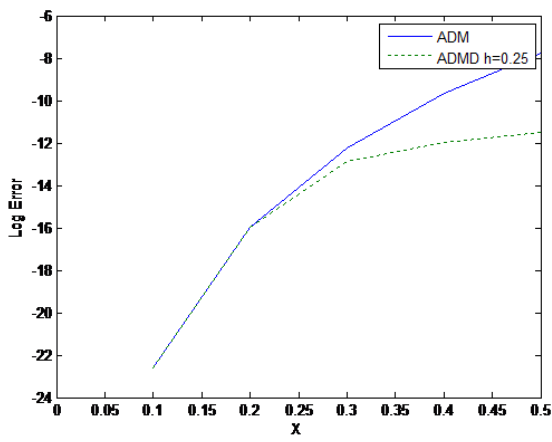

Fig. 4: Comparison between exact solution, solution obtained using ADM and solution obtained using ADMD for $h=0.25$ where Log Error is the logarithm value of absolute error.

TABLE V: Error values for the approximation solution of ADM and ADMD

\begin{tabular}{c||c|c|c}
\hline \multirow{2}{*}{$x$} & \multirow{2}{*}{ Error ADM } & \multicolumn{2}{c}{ Error ADMD } \\
\cline { 3 - 4 } & & $h=0.25$ & $h=0.1$ \\
\hline \hline 0 & 0 & 0 & 0 \\
0.1 & $1.93 \mathrm{e}-16$ & $1.93 \mathrm{e}-16$ & $1.93 \mathrm{e}-16$ \\
0.2 & $1.04 \mathrm{e}-16$ & $1.04 \mathrm{e}-16$ & $9.71 \mathrm{e}-17$ \\
0.3 & $2.18 \mathrm{e}-15$ & $7.91 \mathrm{e}-16$ & $8.33 \mathrm{e}-17$ \\
0.4 & $7.04 \mathrm{e}-14$ & $2.39 \mathrm{e}-15$ & $3.33 \mathrm{e}-16$ \\
0.5 & $1.02 \mathrm{e}-12$ & $2.29 \mathrm{e}-14$ & $0.00 \mathrm{e}+00$ \\
\hline
\end{tabular}

d) Example 4: Consider the following non-linear differential equation

$$
y^{\prime \prime}-6 y^{2}=0 \text { with } y(0)=1 \text { and } y^{\prime}(0)=-2
$$

The exact solution is $y(x)=(1+x)^{-2}$. The result of ADM and ADMD are illustrated in Table IV below with five terms of the series at $h=0.25$. Clearly, ADMD is more accurate than ADM. Figure 4 shows the comparison between ADM and ADMD at $h=0.25$.

e) Example 5: Consider the following non-homogeneous linear differential equation

$$
y^{\prime \prime}-y=2 \text { with } y(0)=0 \text { and } y^{\prime}(0)=0
$$

The exact solution is $y(x)=e^{x}+e^{-x}-2$. The result of ADM and ADMD are illustrated in Table $\mathrm{V}$ below with five terms of the series at $h=0.1$ and $h=0.25$. Clearly, ADMD is more accurate than ADM. Figure 5 shows the comparison between $\mathrm{ADM}$ and ADMD at $h=0.1$ and $h=0.25$. 


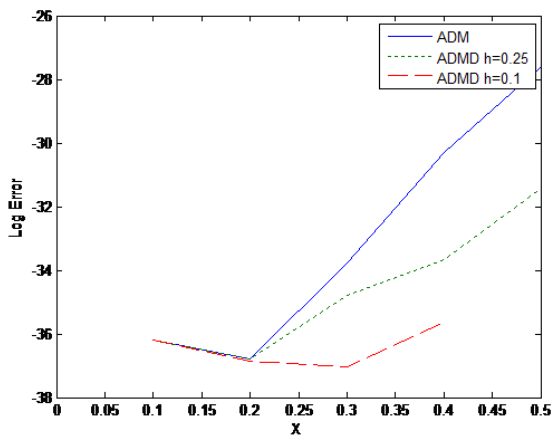

Fig. 5: Comparison between exact solution, solution obtained using ADM and solution obtained using ADMD for $h=0.25$ and $h=0.1$ where $\log$ Error is the logarithm value of absolute error.

\section{CONCLUSIONS}

In this paper, the comparison ADM and ADMD methods for solving second order linear and nonlinear initial value problems is presented. The numerical efficiency of the ADM and ADMD methods is tested by considering five examples. The results show that the ADMD is more effective and accurate than ADM. The accuracy of the ADMD can be improved by taking small step size or by adding more terms of the solution series.

\section{REFERENCES}

[1] G. Adomian, "A review of the decomposition method in applied mathematics," Journal of mathematical analysis and applications, vol. 135, no. 2, pp. 501-544, 1988.

[2] K. Al-Khaled and M. Anwar, "Numerical comparison of methods for solving second-order ordinary initial value problems," Applied Mathematical Modelling, vol. 31, no. 2, pp. 292-301, 2007.

[3] A. Elsaid, "Adomian polynomials: A powerful tool for iterative methods of series solution of nonlinear equations," Journal of Applied Analysis and Computation, vol. 2, no. 4, pp. 381-394, 2015.

[4] M. Al-Mazmumy, A. Al-Mutairi, and K. Al-Zahrani, "An efficient decomposition method for solving bratu's boundary value problem," American Journal of Computational Mathematics, vol. 7, no. 01, pp. 84-93, 2017.

[5] G. Nhawu, P. Mafuta, and J. Mushanyu, "The adomian decomposition method for numerical solution of first-order differential equations," Journal of Mathematical and Computational Science, vol. 6, no. 3, pp. 307-314, 2016.

[6] E. Agom, "Application of adomian decomposition method in solving second order nonlinear ordinary differential equations," International Journal of Engineering Science Invention, vol. 4, pp. 60-65, 112015.

[7] H. Alkresheh, "New classes of adomian polynomials for the adomian decomposition method," International Journal of Engineering Science Invention, vol. 5, no. 3, pp. 37-44, 2016

[8] A.-M. Wazwaz, "A comparison between adomian decomposition method and taylor series method in the series solutions," Applied Mathematics and Computation, vol. 97, no. 1, pp. 37-44, 1998.

[9] J. Edwards, J. Roberts, and N. Ford, "A comparison of adomian's decomposition method and runge kutta methods for approximate solution of some predator prey model equations," 1997.

[10] K. Abbaoui and Y. Cherruault, "Convergence of adomian's method applied to differential equations," Computers \& Mathematics with Applications, vol. 28, no. 5, pp. 103-109, 1994.

[11] _ _ "Convergence of adomian's method applied to nonlinear equations," Mathematical and Computer Modelling, vol. 20, no. 9, pp. 69-73, 1994. 